\title{
Mapping between VI models of orientation selectivity: From a distributed multi-population conductance-based refractory density model to a firing-rate ring model
} Anton V Chizhov*1,2, Elena Yu Smirnova ${ }^{1}$ and Lyle J Graham²

\author{
Address: ${ }^{1}$ Ioffe Physico-Technical Institute of RAS, St. Petersburg, Russia and ${ }^{2}$ Lab. of Neurophysics, CNRS UMR8119, Université Paris Descartes, \\ Paris, France \\ Email: Anton V Chizhov* - anton.chizhov@mail.ioffe.ru \\ * Corresponding author
}

from Eighteenth Annual Computational Neuroscience Meeting: CNS*2009

Berlin, Germany. 18-23 July 2009

Published: 13 July 2009

BMC Neuroscience 2009, I0(SuppI I):PI8I doi:I0.II86/I47|-2202-I0-SI-PI8I

This abstract is available from: http://www.biomedcentral.com/I47I-2202/I0/SI/PI8I

(C) 2009 Chizhov et al; licensee BioMed Central Ltd.

To study the selectivity of neurons in primary visual cortex to the orientation of visual stimuli, we have built a detailed model considering neuronal populations distributed along the cortex and receiving a thalamic input according to a classic pinwheel architecture. The model is based on the refractory density (RD) approach developed in $[1,2]$ for a population of conductance-based adaptive neurons, where "population" implies an infinite number of similar neurons receiving a common input with an implicit noise term that is distinct for each neuron. While in its full form this type of model can be compared with experimental intracellular recordings, for mathematical analysis of the dynamics of feature selectivity the model must undergo considerable reductions. To this end we have constructed a hierarchy of reduced models, at one extreme arriving at the classical firing-rate (FR) ring model [3]. Specifically, we 1) simplify the conductance-based adaptive and fast spiking neuron models to leaky integrate-and-fire neurons, 2) approximate pinwheel architecture by a ring, 3) replace realistic synaptic kinetics by instantaneous kinetics, 4) reduce the number of neuronal populations to one, 5) replace the refractory density approach by the Kolmogorov-Fokker-Planck (KFP) equation describing voltage phase space, 6) substitute the KFP by an artificial relaxation equation for the firing rate driven by its stationary dependence on the input conductance and current ( $f$-g-I-function), and 7) approximate the $f$-g-I-function by a threshold linear $f$-I-curve, thus arriving to the classical FR ring model as described in [3]. With the appropriate analytical expressions we map the parameters of the various models to the four parameters of the FR ring model. We compare the behaviors of the models with the parameter sets corresponding to different domains on a two-parameter plane of the FR ring model corresponding to the intracortical connection strengths. For a range of parameter sets, the comparison shows a consistency for steady-state solutions, whereas the models derived from the probability density approach (RD and KFP) possess a larger variety of solutions, similar to that of the FR ring model with a delay [4]. We then compare the transient solutions of the models when the stimulus orientation changes abruptly. The transient behavior of the $\mathrm{RD}$ and KFP-based models differs significantly from that of the FR model, showing a faster reaction of the cortex. The results of the comparison demonstrate that the constructed hierarchy of models can serve as a useful instrument for fitting mathematical models to experimental data, and their subsequent analysis.

\section{Acknowledgements}

The work is supported by the French-Russian grant PICS CNRS-RFBR-0704-92167 to AVC and LJG, and an Agence Nationale de Recherche grant (FUNVISYNIN) to LJG. 


\section{References}

I. Chizhov AV, Graham LJ: Efficient evaluation of neuron populations receiving colored-noise current based on a refractory density method. Phys Rev E 2008, 77:011910.

2. Chizhov AV, Graham LJ: Population model of hippocampal pyramidal neurons, linking a refractory density approach to conductance-based neurons. Phys Rev E 2007, 75:011924.

3. Hansel D, Sompolinsky $\mathrm{H}$ : Modeling feature selectivity in local cortical circuits. In Methods in neuronal modeling: From synapses to networks 2nd edition. Edited by: Koch C, Segev I. Cambridge, MA: MIT Press; 1998:499-567.

4. Roxin A, Brunel N, Hansel D: Role of delays in shaping spatiotemporal dynamics of neuronal activity in large networks. Phys Rev Lett 2005, 94:238103.

Publish with Bio Med Central and every scientist can read your work free of charge

"BioMed Central will be the most significant development for disseminating the results of biomedical research in our lifetime. "

Sir Paul Nurse, Cancer Research UK

Your research papers will be:

- available free of charge to the entire biomedical community

- peer reviewed and published immediately upon acceptance

- cited in PubMed and archived on PubMed Central

- yours - you keep the copyright

Submit your manuscript here:

http://www.biomedcentral.com/info/publishing_adv.asp 